\title{
Zusammenfassung und Ausblick
}

Vorgestellt wurden akustische Verfahren und die zugehörige Gerätetechnik, um Fragestellungen zu raumklimatologischen und energetischen Problemen untersuchen zu können. Aus der Messung verschiedener physikalischer Parameter wie Schallgeschwindigkeit oder Schalldruck können Aussagen zu Verteilungen von Raumtemperatur und Raumluftströmung oder zu flächenhaften Darstellungen akustischer Undichtheiten, hier gleichgesetzt mit potenziellen energetischer Verluststellen, abgeleitet werden.

Die in den Räumen einzusetzende Messtechnik wird betrieben, ohne dass Eingriffe in die Struktur der Räume notwendig sind. Die Raumklimaverhältnisse selbst werden durch die akustischen Verfahren nur minimal bzw. überhaupt nicht beeinflusst. Das ermöglicht eine nachträgliche Installation oder einen Einsatz beispielsweise in denkmalgeschützten Bauten, wo räumliche Veränderungen nicht gern gesehen sind.

Die vorgestellten Methoden nutzen aktive Schallquellen, um Aussagen zum Raumklima oder zur energetischen Dichtheit von Fenstern, Türen, von Lüftungsschächten und Kabeldurchführungen zu erhalten. Die Größe der erfassbaren Prüfobjekte hängt dabei von Parametern wie Anregungsfrequenz und Leistung dieser Schallquellen ab.

Die vorgestellten Methoden nutzen einen großen Frequenzbereich - von hörbaren bis zu Ultraschallfrequenzen. Vorteilhaft dabei: es ist möglich aus dem Hörschallbereich in den unhörbaren Ultraschallbereich auszuweichen ohne dass sich die Physik und die darauf aufsetzenden Schallanalyseverfahren grundsätzlich ändern. Es ist nur sicherzustellen, dass der Empfänger die ,Musik des Senders hören und verstehen kann. Die Personen in der Nähe der Messtechnik nehmen dann diesen Schall nicht mehr wahr und man umgeht das Problem der Akustiker das schon Wilhelm Busch 1872 so prägnant auf den Punkt brachte „Musik wird oft nicht schön gefunden, weil stets mit Geräusch verbunden“".

(C) Der/die Autor(en), exklusiv lizenziert durch Springer Fachmedien

Wiesbaden GmbH, ein Teil von Springer Nature 2021

A. Raabe und P. Holstein, Akustik und Raumklima, essentials, https://doi.org/10.1007/978-3-658-33324-9_6 